\title{
VIRTUAL REALITY IN SECOND LANGUAGE ACQUISITION RESEARCH: A CASE ON AMAZON SUMERIAN
}

\author{
Ana Maria Vieira Monteiro ${ }^{* 1}$ and Thies Pfeiffer ${ }^{2}$ \\ ${ }^{1}$ Universidade Federal de Juiz de Fora, Rua José Lourenço Kelmer, s/n - Bairro Martelos. Juiz de Fora - MG, Brazil \\ ${ }^{2}$ University of Applied Sciences Emden/Leer, Constantiaplatz 4, 26723 Emden/Leer, Germany
}

\begin{abstract}
Virtual reality (VR) has gained increasing academic attention in recent years, and a possible reason for that might be its spread-out applications across different sectors of life. From the advent of the WebVR 1.0 API (application program interface), released in 2016, it has become easier for developers, without extensive knowledge of programming and modeling of 3D objects, to build and host applications that can be accessed anywhere by a minimum setup of devices. The development of WebVR, now continued as WebXR, is, therefore, especially relevant for research on education and teaching since experiments in VR had required not only expertise in the computer science domain but were also dependent on state-of-the-art hardware, which could have been limiting aspects to researchers and teachers. This paper presents the result of a project conducted at CITEC (Cluster of Excellence Cognitive Interaction Technology), Bielefeld University, Germany, which intended to teach English for a specific purpose in a VR environment using Amazon Sumerian, a web-based service. Contributions and limitations of this project are also discussed.
\end{abstract}

\section{KEYWORDS}

Virtual Reality, Second Language Acquisition, Vocabulary, WebXR, Amazon Sumerian

\section{INTRODUCTION}

By naming an experience VR, we refer at least to a visualization in 360 degrees of digital content displayed, e.g., inside a visor, called virtual reality headset or HMD (Head Mounted Display). Such a device fits on the head of its users in a way to block or minimize the interference with the surrounding environment. Moreover, some of these devices allow 6 degrees of freedom (meaning the possibility of moving right and left, up and down, forward and backward, combined with rotation on three perpendicular axes) and the manipulation of objects in the virtual world by adding controllers to the hands of its users.

The gaming industry has been taking the lead on the development of consumer VR technology and applications, but many other markets have shown interest in it as an ultimate tool, education included, especially in the field of hard sciences, where some concepts gain a new perspective when they can be perceived in 3D and 360 degrees (Curcio, Dipace \& Norlund, 2016). In addition to this trend, which focuses on problem-solving, simulations of scenarios and historical events are also found, where the user receives information in the form of text and/or audio while contemplating the virtual environment. Among the vast possibilities in the field of education, this paper is particularly concerned about WebVR as a means for second language acquisition (SLA). More specifically, how immersion can be beneficial for vocabulary learning. By immersion, it is understood here the techniques that enable the user of a system to feel an illusion of non-mediation between them and the virtual world. VR environments are experienced in the first-person perspective, and such a perspective might be worth investigating since it is easier to translate foreign language vocabulary when autobiographic memories take part in its evoking, i.e., when a person is asked to translate a foreign word which has a significant contextual meaning in their lives (Matlin, 2004).

Although the research interest on VR has been noted for some time in the CALL field (Schwienhorst, 2002), only recently it became possible to create web-based life-sized experiences with natural interactivity.

\footnotetext{
${ }^{*} \mathrm{CNPq}$ scholar.
} 
Moreover, academic projects towards VR in the field of foreign language vocabulary learning are scarce, and a possible reason for that had been the restricted access to the technology in Humanities before WebVR became a suitable tool. As the literature review shows, by the time this research started, there were only a few comparative studies about this topic (Cho, 2018; Vazquez et al., 2018; Gupta, 2016). Meanwhile, VR has been used commercially, in the field of language instruction, mostly as training of ready-made sentences for travellers. Therefore, one could ask if VR is a replacement for classroom role-plays and drills, as well as what would take for the learner to feel immersed conversationally in the virtual space.

The following topics introduce how Amazon Sumerian, an online platform for the development of augmented and virtual reality applications based on WebVR/WebXR, can be of use in the SLA research field, considering the two aspects mentioned above, as well as its limitations within Amazon Web Services.

\section{VOCABULARY AND COMMUNICATION IN CONTEXT}

During a research visit of Ana Monteiro at CITEC's virtual reality group led by Thies Pfeiffer, now Professor at Emden, a group project was created, entitled Second language vocabulary learning for health professionals. This project had the support of the Nursing Faculty at an institution nearby, where other projects had been conducted before to train the Nursing students certain procedures in VR. The main work during the summer semester of 2019 was to build scenes in VR so they could be a context-based scenario for learning English vocabulary related to wound care materials and for the practicing of protocols for good and effective communication between the nurse and the patient. Besides labeling the target-objects with name tags that would appear when the player touches them in VR, a dialogue system (outside AWS) using both text and voice input was built, creating a bi-lingual communication between the user and the guide of the experience (a virtual agent). In this way, the player could ask for translations in either English or German.

\subsection{Amazon Sumerian}

Amazon Sumerian (hereafter AS) is one of the services offered by AWS. It allows users to create and publish AR and VR scenes via an URL accessible from any compatible browser (the latest versions of Chrome or Firefox), which is made possible via the WebVR standard. AS supports the main VR headsets in the market today, including the now open-sourced Google Cardboard. The HTC Vive headset and controllers were chosen for this project, for its higher quality.

To begin developing with AS, an account on AWS must be created. This account gives access to other services such as Amazon Lex and Amazon Polly, which were also used in our prototype, and it can be free for educational purposes. Programming knowledge is not needed since AS uses an editing system based on a visual representation of coding called state machines. However, as the developer's goals become more specific, knowledge of JavaScript allows connecting services outside AWS' scope which can be useful for a system including natural language processing (NLP), as we exemplify below. AS allows the user to import 3D objects, together with their materials and animations, from modeling programs (such as Blender, a free, open-source software) and online assets shops. AS also features the so-called hosts, human-like characters that can guide the user through the experience via speech recognition and text input.

\subsubsection{Models and animations}

Since the 3D objects available in the AS library did not correspond entirely to the needs of the scene in the project, two students were responsible for modeling a few elements in Blender and export them to AS. During this process, we realized there were some limitations on the size of the images regarding texture, as well as a limit of vertices AS can render. Despite that, two scenes were composed with all the objects and respective animations needed for the simulations of a wound care procedure. Relevant to note, however, is that the scene in VR cannot function properly with as many objects and textures as the desktop version of the same scene. Thus, as the model for the host could not be implemented beyond scene 01 , we kept only its voice acting as the player's guide. 
For acting as a patient (Figure 1), another character had to be made outside AS since hosts cannot have their bodies and vertical position changed. As a solution, the students used two other software products, Fuse, for character building, and Mixamo, for character animation (both from Adobe and free to use in the context of this project).

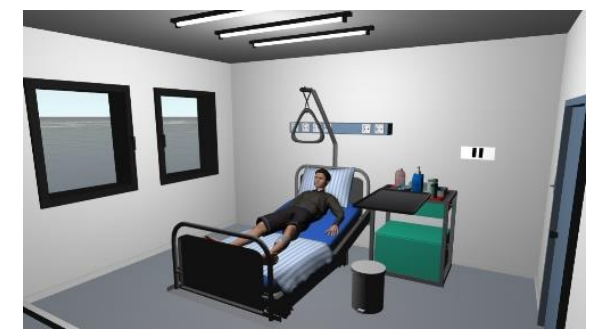

Figure 1. Patient rigged in Mixamo, which added a ready-made animation for lying down and breathing

\subsection{Vocabulary Teaching Methods}

As a prototype, this project sought to experiment with both explicit and implicit methods of teaching words and phrases in context. One was by directly showing the player the names of the objects they were manipulating. The other one was via the dialogue component of the scene, meaning the voice interactions the player can have with either the guide or the patient. While talking to the patient, as good practice, the nurse must be able to explain what they are doing and that is where the name labels on the objects become useful. Repetitive exposure to the name of the items, regarded as crucial in vocabulary learning (Koda, 2012), comes in naturally at play since the same objects are manipulated more than once.

\subsubsection{The Dialogue}

AWS features a chatbot system called Lex, which could not be used for the wanted interactions in the project because of its limitations for dialogue flow. For example, it requires intents, sentences that are likely to be said by the player. Since we wanted to build a system that dealt with a degree of unpredictability in the sentences spoken by the player, another option was found outside AWS, on Wit.ai, a free NLP resource for web-developers. Incorporating such a tool to AS is possible, but not through the visual interface and therefore knowledge of JavaScript was needed. We used keywords so the player does not get frustrated if they do not remember or say correctly an entire English sentence. Moreover, Wit.ai made possible the switch between two languages (German and English), which could be accessed by a specific triggering sentence directed to the virtual guide (Maya), such as in Maya, can you translate that?

The dialogue was segmented in 10 units concerning different parts of communication skills for the interaction nurse-patient, e.g., explanation of procedures and small-talk. If the player says something that is not expected, the virtual guide interferes in the dialogue to give the player a hint. After each segment is done correctly, two buttons appear in the scene, one to continue, and go to the next section of the dialogue, and another one to redo that dialogue section. The player can also pause the scene at any time.

Finally, to give the characters different English variations, we used Amazon Polly, a text-to-speech application that can be managed directly from the AS console. Polly also allows the customization of these voices in terms of prosody, enabling the characters to sound closer to a human voice.

\section{NEXT STEPS}

A comparative study will be applied to Nursing students at Universidade Federal de Juiz de Fora, after the ongoing English for specific purposes module being taught by Ana Monteiro in the same institution. This study will expose the participants, divided into two groups (control and experimental), to the desktop and VR conditions for learning the target-words related to wound care. For the experimental group in the VR condition, HTC Vive headsets and controllers will be used. 
The quantitative data of the experiment, i.e., the pre and post-vocabulary tests, as well as the qualitative data, obtained from questionnaires, will be analyzed to answer these questions: 1) Does VR contribute for short and long-term recall of English vocabulary as a second language?, and 2) To what extent does the affective component influence English vocabulary learning in VR?

The fact that AS is web-based is something to consider if the results obtained from the experiment are promising for education purposes. That means it will be easier for other researchers and teachers to replicate the methodology of the study, once there is no need for high-end computers and headsets.

\section{CONCLUSION}

Language and cultural differences can be a barrier to ensure best-as-possible care for patients, as it is the case in German hospitals, where many health professionals are often dealing with foreign language patients. Language learning for specific purposes has in virtual reality a combination of a more realistic environment, task performance training, voice recognition and feedback of virtual agents. For researchers in the SLA realm, the possibilities offered by WebVR resources have the potential to expand experiences such as the one presented here to other fields where language training is combined with specific tasks. For learners, a VR environment can be a practicing field for reducing the anxiety of speaking in the real world. For institutions, a VR language training program can be a low-cost option for professionals' continuing education. In sum, WebVR represents not only an alternative to common traditional practices of second language classes but it can offer, to a certain extent, a real feel of participation in a life-like, less predictable conversation. Acquiring the programming knowledge for that to happen is, perhaps, the biggest limitation imposed on an Applied Linguistics researcher who wishes to build such a system. Nonetheless, since Applied Linguistics has been essentially an interdisciplinary field, the challenge can be overcome by cooperation with other areas of knowledge such as Computer Science, as showcased in the frame of this work.

\section{ACKNOWLEDGMENT}

We would like to thank the collaborators at CITEC, as well as the students from Bielefeld University who helped us to build the VR environment: Anila Dawar, Felix Dyck, Jennifer Bitschene, Michael Gröning, Morten Biermann, Muhammad Katri, Teena Chikaral, and Tim Heptner.

\section{REFERENCES}

Cho, Y. (2018). How Spatial Presence in VR Affects Memory Retention and Motivation on Second Language Learning: A Comparison of Desktop and Immersive VR-Based Learning (Unpublished master's dissertation). Syracuse University, Syracuse, New York.

Curcio, I. D. D., Dipace, A., \& Norlund, A. (2016). Virtual realities and education. Research on Education and Media, 8(2), 60-68. https://doi.org/10.1515/rem-2016-0019

GUPTA, S. (2016). OGMA: language acquisition system using immersive virtual reality (Unpublished master's dissertation). The University Of Texas At Arlington, Arlington, Texas.

Koda, K. (2012). Development of second language reading skills: cross-linguistic perspectives. In S. Gass \& A. Mackey. The Routledge Handbook of Second Language Acquisition. (p. 303-318) New York: Routledge.

Matlin, M. (2004). Psicologia Cognitiva. Rio de Janeiro: LTC.

Schwienhorst, K. (2002). The state of VR: A meta-analysis of virtual reality tools in second language acquisition. Computer-Assisted Language Learning, 15 (3), 221-239.

Vazquez, C., Xia, L., Aikawa, T., \& Maes, P. (2018). Words in Motion: Kinesthetic Language Learning in Virtual Reality. 2018 IEEE 18th International Conference on Advanced Learning Technologies (ICALT). Presented at the 2018 IEEE 18th International Conference on Advanced Learning Technologies (ICALT). https://doi.org/ 10.1109/icalt.2018.00069 